\title{
ABRIR LAS CIENCIAS SOCIALES
}

\section{Immanuel Wallerstein Traducción de Fernando Cubides}

\section{Nota introductoria}

\begin{abstract}
Las páginas siguientes constituyen el registro de las palabras pronunciadas por Immanuel Wallerstein el 24 de octubre de 1995 en la Social Science Research Council de Nueva York. Su objetivo era la presentación del volumen Open the Social Sciences, un informe sobre la reestructuración de las ciencias sociales auspiciado por la Comisión Gulbenkian. Wallerstein es profesor de la Universidad del Estado de Nueva York en Binghamton y tiene a su cargo la dirección del Centro Fernand Braudel dedicado al estudio de la economía, las civilizaciones y los sistemas históricos. La editorial siglo XXI de México ha difundido en español los dos primeros volúmenes de su extensa obra El moderno sistema mundial, que acaba de ser escogido por la revista Contemporary Sociology como uno de los diez libros de ciencias sociales más influyentes en los últimos 25 años. El libro desarrolla la teoría de la economíamundo, un influyente y ambicioso marco de referencia de la sociología histórica norteamericana que estudia el impacto del capitalismo en la civilización moderna, El texto de esta presentación apareció originalmente en Items, el boletín del Social Scíence Research Council (vol, 50:1, marzo de 1996).G.C.
\end{abstract}

¿Cómo se construyeron las Ciencias Sociales? Al preparar nuestro informe tuvimos que considerar este asunto para entender los dilemas que ellas confrontan. Comenzamos el relato a fines del siglo XVII1 anotando que el más importante acontecimiento de la época fue una especie de divorcio definitivo —dudo al usar la palabra "divorcio"-, una ruptura entre la ciencia y la filosofía.

Anteriormente, los vocablos que las designaban, si no eran del todo intercambiables, estaban imbricados de modo muy directo. Ambos significaban conocimiento y la gente no establecía una nítida distinción entre filosofía y ciencia. A fines del siglo de las luces asistimos al nacimiento de lo que C. P. Snow denominó "las dos culturas". La ciencia comenzó a definirse por su contenido empírico, a ser entendida ante todo como una búsqueda de la verdad a través de la investigación, a diferencia de lo que estaban haciendo los filósofos, especular o deducir de algún modo. Fue una continuación de la ruptura entre la filosofía y la teología; aquí se daba un paso más hacia un sistema de conocimiento íntegramente secularizado.

\section{La Universidad y el conocimiento}

Al tiempo que se producía la fisura intelectual entre la filosofía y la ciencia en la mentalidad de la época, se operaba un resurgimiento de la universidad. Solemos hablar de la universidad como una institución continua, pero ello no es del todo cierto. La universidad medieval fue una institución muy interesante, pero prácticamente había muerto a fines del siglo XVI. Y las universidades llegan a ser insignificantes a lo largo de los siglos XVI, XVII y XVIII. Carecían de un cuerpo directivo permanente, y lo esencial del trabajo intelectual se llevaba a cabo al margen de ellas y en otro tipo de instituciones como el Collège de France, o la Royal Society. Una de las cosas realmente interesantes

\footnotetext{
*Profesor del Departamento de Sociología de la Universidad Nacional de Colombia.
} 
que ocurren en el siglo XIX es la reinvención de la universidad como el ámbito, tanto de la creación del conocimiento como de su reproducción. Ello trajo algo nuevo que afectaría a las Facultades, la de Filosofía al comienzo, y las desintegraría hacia algo que posteriormente se denominaría las disciplinas, dotadas de cátedras con departamentos que otorgaban títulos académicos. La estructura de la universidad tal como la conocemos hoy se creó en verdad a fines del siglo XIX; por lo tanto la universidad y las disciplinas que la conforman constituyen una invención muy reciente.

En términos del desarrollo de las disciplinas individuales, aproximadamente entre más o menos 1750 y 1850 nos hallamos en una situación en la que surgen centenares de nombres para los campos de investigación. Pero entre 1850 y 1914 asistimos a la reducción de estos nombres a un pequeño número de denominaciones que al final se convierten en las disciplinas. Esto se lleva a cabo mediante una especie de coagulación de conjuntos de intereses, conjuntos de problemas. Nuestro informe arguye que mediante tal agregación arribamos a los seis grandes nombres corrientes hoy en día, más un par de nombres menores. Estas seis grandes denominaciones se convierten en departamentos, en asociaciones profesionales, en revistas académicas y en sistemas de clasificación en las bibliotecas (la Biblioteca del Congreso de los Estados Unidos, por ejemplo, las reproduce como conjunto de categorías en la última década del siglo XIX). Todo ello institucionaliza una serie de opciones.

\section{Líneas de demarcación}

Podemos considerar dichas denominaciones con referencia a tres criterios básicos de delimitación. El primero es el de pasado-presente, que configuró una neta diferenciación entre la historia, tal como se reorganizó en el siglo XIX, y el trío conformado por la sociología, la ciencia política y la economía. Se conforman dos presupuestos bien diferenciados acerca de cómo se llega a la verdad científica. Los historiadores siguen la sentencia de Ranke de que "er will blo $\beta$ zeigen, wie es eigentlich gewesen" (tratamos, simplemente, de exponer cómo ocurrieron en realidad las cosas). En otras palabras, se debería tomar como evidencia los documentos escritos en la época en cuestión, en particular aquellos que fueron escritos con propósitos distintos dc los de informar al historiador de tres siglos después. La asunción fundamental es que si un embajador le escribe una carta a su monarca, él está tratando de informarle acerca de la situación del país a donde ha sido asignado, y lo está haciendo tal y como ha llegado a entenderla. Si usted lee ese informe tres siglos después, llega a saber en últimas lo que dijo el embajador, que bien puede ser lo que realmente el embajador creía. Y ello a su vez lo impulsa a usted hacia unos determinados archivos. Y también, claro está, en dirección a la historia política y diplomática, que es por cierto la parte de la historia que suele estar mejor conservada en los archivos.

Se ha postulado que los académicos suelen incurrir en prejuicios acerca de los hechos de su tiempo a causa de que están involucrados en sus propias sociedades. Por tal razón, mientras más atrás se remonten en la historia, más neutrales podrían ser. Además, la realidad objetiva del archivo se impone en el estudioso; no hay allí nada que sea reciente y por ende sospechoso. Por su parte, tanto los Estados como las demás instituciones, no suelen facilitar el acceso a sus documentos recientes a los investigadores. Todavía hoy los documentos de Estado son secretos por cerca de 20, 30, 50, 100 años o aún más. Por lo demás, con el fin de entender los archivos, se debe estar bastante bien informado acerca del contexto cultural en el que se ubican. Esto suele llevar a los historiadores a trabajar en los campos que les resultan más familiares, y de allí la tendencia preponderante a trabajar en las historias de sus propios países. A la vez se hallan 
bastante predispuestos contra las generalizaciones, precisamente porque son "científicos". Es decir, tienden a ver las generalizaciones como anticuadas, propias de la vieja filosofía especulativa, y si usted quiere ser empírico no debería generalizar. En cualquier caso trabajar con archivos lo lleva a ser más y más detallado, y sabemos que los detalles tienden a ser terriblemente ideográficos.

Por su parte el trío nomotético tiene a su vez toda la lógica en su cabeza. Para ser objetivo, dicen, debemos aportar ante todo datos que no estén al arbitrio del investigador. Mientras más cuantitativos sean los datos, menos dependen de la subjetividad del estudioso y más comparables son en situaciones diversas. Tal postulado nos conduce inexorablemente al presente. Especialmente si se da el paso siguiente que es postular que hay verdades universales acerca de la conducta humana que atraviesan todo tiempo y todo espacio. Al minuto de decir eso, desaparece la diferencia de estudiar la Alemania de hoy o la India del siglo y antes de nuestra era, puesto que se buscan verdades universales. En tanto que los datos sobre la Alemania de hoy son 5000 veces mejores - 0 podríamos decir pesan 5000 veces más- que los datos de la India de aquel periodo, terminamos estudiando a Alemania con el fin de arribar a nuestras generalizaciones. Por lo general esa solía ser la separación que se daba a fines del siglo XIX y a comienzos del actual entre la historia y las tres ciencias sociales "duras".

Debemos acotar algo más: la sociología del conocimiento. Por lo menos el 95\% de todos los estudiosos y de la investigación académica en el período entre 1850 y 1914 , y probablemente hasta 1945, proviene tan sólo de cinco países: Francia, Gran Bretaña, las Alemanias, las Italias y los Estados Unidos. Hay todavía algo más; no sólo la investigación académica proviene de estos cinco países, sino que gran parte de la investigación hecha por la mayoría de los estudiosos es sobre su propio país. Esto es parcialmente pragmático y en parte obedece a presión social y a razones ideológicas: son los países importantes, lo que interesa y lo que debemos estudiar si queremos saber cómo opera el mundo.

Esto nos conduce a la segunda diferenciación. El hecho real es que los cinco países en cuestión no eran el mundo entero, y había en la comunidad académica la vaga sospecha de que había un mundo más alejado de ellos. Lo que se hizo, a nuestro juicio, fue inventar dos disciplinas adicionales que abarcaran el resto del mundo. La primera y más obvia fue la antropología, a la que se creó para estudiar el mundo primitivo. Al mundo primitivo se lo define de un modo bastante simple: en la práctica vienen a ser las colonias de los cinco países anotados, incluyendo a su frontera interna. Teóricamente, su objeto se puede definir como aquellos pequeños grupos de bajo nivel tecnológico que carecían de escritura antes de sus contactos con Occidente y que no tenían creencias religiosas que fueran más allá del propio grupo —cada uno tenía sus creencias características. Se presumía que estaban estancados y el tiempo no transcurría para ellos.

En fin tenemos toda una ideología acerca de cómo abordarlos. Son gente muy extraña, que habla lenguas bastante raras desde el punto de vista europeo. Tenía entonces que irse allí, hacer observación participante, permanecer un par de años con "su tribu", aprender el idioma consiguiendo que alguien haga de intérprete. ¿Y qué se estudia? Pues todo: etnografía. Puesto que para comenzar no sabemos nada, se ha de aprender todo: cómo se casan, cómo intercambian bienes, cómo ventilan sus diferencias, cuál es la gramática de la lengua, y al regreso hacemos un detallado informe de todo esto. Era algo bastante ideográfico, basado además en la presunción de ahistoricidad. 
Esto nos ayudaba a resolver el problema de una gran parte del mundo, pero no de todo el mundo, puesto que obviamente había un grupo de países que no encajaban para nada en los anteriores parámetros, que no podrían ser descritos en los términos que he utilizado para describir el trabajo antropológico: China, India, el mundo árabe, Persia. Todos ellos comparten un conjunto de características. Tienen en la actualidad, o tuvieron en algún momento del pasado, uno o más grandes imperios burocráticos en su territorio. Como resultado de ello tienen escritura y múltiples textos que se han preservado. Además, todos ellos tienen - para usar una expresión del siglo XIX - "religiones mundiales". El término religión mundial significa esencialmente que se trata de una religión que se ha propagado a una vasta extensión del mundo. El budismo, el islam y el hinduismo son religiones mundiales por oposición a muchas creencias religiosas del Africa que comparten un animismo muy localizado. Tales civilizaciones no europeas poseen religiones mundiales y tienen textos que tienden ampliamente a ser textos religiosos. Lo único que no tenían era modernidad.

El estudio de esta clase de sistemas sociales se fue construyendo en un último campo al que no se lo definía propiamente como ciencia social, pero que de hecho era la ciencia social más amplia al ocuparse de todas esas áreas del mundo: los estudios orientales. La premisa de estos estudios era bastante simple: eran estructuras maravillosas, complejas que deberíamos comprender. La mejor forma de hacerlo era penetrando en su civilización, lo que en principio significaba leer y aprender los textos —la filología llegó a ser una técnica de gran importancia- y presentarlos al resto del mundo, mientras se explicaba porqué no habían llegado a ser modernas. Tendieron a ser vistas como civilizaciones congeladas y por lo tanto ahistóricas. Con lo cual tenemos configurada esa segunda delimitación básica: la historia más el trío nomotético dirigido al mundo occidental, y la antropología y los estudios orientales relacionados con el resto del mundo.

La tercera de las delimitaciones tiene que ver con la existencia de las tres ciencias sociales nomotéticas (la sociología, la ciencia política y la economía) ¿Por qué no una única ciencia social? Pienso que la respuesta tiene que ver con la ideología dominante a lo largo del siglo XIX. Básicamente, el punto de vista dominante a nivel mundial del liberalismo, era que el estado, el mercado y la sociedad eran tres entidades diferenciadas. Ellas operaban con lógicas diferentes y por lo tanto debían ser estudiadas en forma separada, y en cierto sentido, se mantenían aparte en el mundo real. Por eso los estudiosos tenían que segregar su conocimiento de tales aspectos. En líneas generales eso fue lo que pasó, y lo que hacia 1945 estaba ya establecido como principio organizativo para las ciencias sociales en las principales universidades. En el propio surgimiento del sistema universitario como tal tenemos entonces lo que denominaríamos la división tripartita, entre las ciencias naturales, las humanidades y las ciencias sociales. Básicamente, eso es lo que significa filosofía versus ciencia, con las ciencias sociales en algún punto intermedio, reproduciéndose en el interior de éstas la tensión resultante de la contraposición de las "dos culturas". Arribamos así a 1945. Y entonces todo esto cambió.

\section{La internacionalización de las Ciencias Sociales}

Pensamos que todo cambió con posterioridad a 1945, primordialmente porque el mundo real cambió en varios sentidos. Tras la segunda guerra mundial surgen los Estados Unidos como la fuerza dominante económica, política y culturalmente. Por cerca de 10 o 15 años llega a ser de modo literal y numérico dominante también en el mundo de la ciencia social. Yo mismo me sorprendí al examinar uno de los informes de la UNESCO en los años de la posguerra y constatar cómo, de un comité de 16 miembros, 15 provenían de los Estados Unidos. $Y$ hoy no puedo imaginar un documento de la misma UNESCO en el 
cuál de los 16 miembros haya más de uno proveniente de los Estados Unidos. ¿Qué diferencias hay en uno y en otro caso? Podríamos decir que varias, y una de ellas es la presencia de ciertos estudios por áreas geográficas. La historia es muy clara al respecto, y la motivación principal era de índole geopolítica. La gente se decía: "los Estados Unidos tienen todas esas responsabilidades en el mundo y no hay nadie que sepa con precisión qué está pasando en la mayor parte de él; estamos cortos de investigadores, debemos producir especialistas sobre la parte no occidental del mundo". Surgen entonces los estudios de áreas como modo de organización mediante el cual se pueden producir con rapidez gran número de investigadores que llegan a acumular algún saber sobre África, Asia, América Latina, Rusia China y cualquier otra región.

Se trata de una estructura organizativa muy interesante. La idea básica fue algo así como, "no modificaremos la estructura de las disciplinas. La gente seguirá obteniendo sus doctorados (sus Ph.D.) en cada una de ellas; pero trataremos de inducir a los estudiantes de posgrado a que se especialicen en las áreas y adquieran conocimiento sobre ellas dándoles al menos un año más a los requeridos normalmente para su Ph.D., durante el cual pueda aprender un poquito de todo acerca de la región de que se trate". Si se estaba interesado en la India, tenía entonces que aprender algo de historia de la India, de la sociología de la India, de su economía, de su ciencia política. Después podía estudiar el idioma urdu o hindi, o lo que fuese. Esto se llamó - para usar la expresión ya consagrada - estudios multidisciplinarios. Los estudiantes adquirían ese conocimiento, en seguida obtenían su Ph.D. en alguna de las disciplinas y luego se esperaba que continuasen con su trabajo empírico sobre la India ya como sociólogos, como economistas o historiadores. Debe decirse que fue un programa bastante exitoso que en los últimos 40 años se expandió más allá de los Estados Unidos. Muchos países adoptaron el mismo esquema y así es como hemos producido miles de investigadores de primera línea, haciendo toda clase de trabajos, lo que hubiera sido impensable antes de 1945.

¿Pero qué significa esto ahora? Ante todo, que la delimitación mundo civilizado/resto del mundo se ha roto por completo en términos de las disciplinas. Antes de 1945 se hubiese considerado bastante extraño, que usted emprendiera un trabajo empírico por fuera del mundo occidental si no fuera antropólogo u orientalista. $Y$ de pronto aparecen historiadores, politólogos, sociólogos e incluso economistas ocupándose del resto del mundo. Si se reflexiona sobre ello encontraremos que parece haberse subvertido la lógica teorética de la antropología cultural y de los llamados estudios orientales. Dicha lógica consistía en que aquellas disciplinas tenían cosas especiales que hacer en esas áreas que nadie más podría hacer $-\mathrm{y}$ que además debían hacerlo en forma diferente. Y como solían hacerlo de un modo ahistórico, pronto son rebasados al encontrarse con nuevos enfoques, ya se trata de una realidad muy dinámica y cambiante —esa fue la razón por la que se crearon los estudios de áreas. Y ello desafió la lógica de las disciplinas. Los estudios orientales pierden su nombre, los investigadores se unen a otras divisiones y se convierten en historiadores o en profesores de religión. Los antropólogos culturales ensayan varias cosas. Deciden que tanto Europa como Norteamérica también tienen sus propias tribus; se dedican al estudio de los montañeses suizos y de los habitantes de los barrios pobres de Chicago, y en seguida resuelven lo que estudiarán, la "cultura". Están en la búsqueda de una raison d'être.

De modo concomitante ha cambiado la lógica interna de los departamentos. No se trata tan sólo de que la antropología cultural y los estudios orientales hayan perdido su exclusividad, sino también de que otras disciplinas tienen que ocuparse de sus fundamentos racionales y metodológicos. Y por encima de todo, con posterioridad a 1945 
asistimos a la más extraordinaria expansión de la economía mundial en la historia del moderno sistema mundial. A su vez significa que hay una gran cantidad de dinero de por medio, y que una parte importante de ese dinero se ha empleado en la también increíble expansión del sistema universitario a todo lo ancho del mundo. Desde 1945 a esta parte se ha producido un incremento en progresión geométrica en el número de universidades, de profesores universitarios, de estudiantes, de estudios de posgrado...

Cuando miramos nuestros doctorados, hay algo indefinido acerca de lo que se exige como investigación original. Investigación original significa cualquier cosa que hasta el momento no haya sido investigada. $\mathrm{Y}$ como el número de doctorados se incrementa rápidamente, eso tiende a ser un problema. Uno tiene que encontrar sus propios nichos. Se desarrolla entonces un proceso natural de incursiones furtivas. Citaré como ejemplo mi propio campo de estudio, la sociología. Una de las primeras subdisciplinas que se desarrolló con posterioridad a la segunda guerra mundial fue la denominada sociología política. Luego vino la sociología económica y un poco después la sociología histórica. Y no quiero hablar de sociologías más esotéricas, como la sociología del turismo, por ejemplo, pero cada una de ellas a la vez significó una incursión en campos vecinos. Recuerdo mi propia experiencia hace unos 40 años cuando hice la sustentación oral de mi disertación doctoral. Uno de los campos en que me movía era la sociología política, y uno de los jurados me interrumpió para preguntarme: ¿"Cuál piensa Ud. que es la diferencia entre la sociología política y la ciencia política"?, una pregunta que, confieso, no se me había ocurrido antes. Reflexioné y solo atiné a contestar: "Bueno, en verdad no encuentro ninguna" $Y$ todavía hoy no la encuentro. Tenemos pues un problema de yuxtaposiciones que crece día a día.

Por una u otra razón asisto a diferentes congresos académicos de carácter nacional. Una de las cosas que me ha impactado al mirar los programas de esos eventos, es que los títulos de las ponencias se parecen cada vez más, y a decir verdad, de guiarse por sus nombres es muy difícil saber en qué congreso se encuentra uno. Los títulos son los mismos tanto en un congreso de sociología como de antropología, ciencia política o historia. La yuxtaposión es cada día mayor. Esta es la situación desde 1945; los estudios de áreas subvirtieron la lógica de la ciencia social al dividir lo que hasta entonces existía. Las mutuas invasiones también contribuyen a esta situación.

Y entonces, simbólicamente, vino 1968, y con él surgieron dos cosas. Primero que todo, uno de los temas principales del 68 fueron los "pueblos olvidados", que de inmediato se tradujeron en términos académicos: estudios sobre la mujer, sobre las negritudes y una serie más de temas y de nombres antes marginales. Sus portadores y cultores afirmaron: ahora tenemos un sitio definido y legitimo en la estructura académica; querríamos también una línea de publicaciones, programas especiales, fondos de investigación e inclusos doctorados sobre el tema -aun cuando sobre esto último todavía haya ciertos escrúpulos. Lo que podemos ver de todo este proceso, fuerte, con una amplia base social y por lo visto irreversible, es que de hecho nos estamos moviendo en otra dirección. Si entre 1750 y 1850 teníamos muchos nombres que después se redujeron a seis en 1945, la curva se está moviendo ahora en dirección contraria. Vamos de seis a veinte nombres. Cuando leo los catálogos universitarios me impresiona que agrupan sus áreas del saber bajo diez o doce denominaciones. Todas las universidades conservan los seis o siete ya consagrados, pero cada una le agrega a su vez tres o cuatro que además varían según la institución de que se trate. Y lo previsible es que esa tendencia continuará en el futuro. 


\section{Esferas de investigación}

En los años setenta y ochenta sucedieron otras dos cosas fundamentales para el tema considerado en este informe. La primera consistió en una revolución de grandes alcances en las ciencias naturales. Las ciencias naturales fueron epistemológicamente muy estables desde el siglo XVII hasta los 70's en el sentido de que las premisas newtonianas y cartesianas siguieron siendo fundamentales para toda la actividad científica. La ciencia siguió considerándose como la búsqueda de las leyes más simples; la ciencia era objetiva, neutral, se ocupaba de los equilibrios y se la consideraba acumulativa.

En verdad esta revolución maduró a finales del siglo XIX, pero ella no adquirió fuerza organizativa sino hasta el decenio de 1970. Viene y nos dice que la ciencia no es determinista y que todo lo que podemos alcanzar es una serie de afirmaciones probabilísticas acerca del futuro. Que la exactitud matemática es imposible de obtener y que cada vez que medimos, se mide algo diferente. Los procesos no son lineales sino bifurcados, que la ciencia es la búsqueda de lo complejo y no de lo simple, y lo que es más importante para nuestro propósito, las leyes científicas son irreversibles. Un presupuesto básico para la ciencia natural era que el tiempo no afectaba la operatividad de la ley. Hoy en cambio varias ciencias naturales proclaman que la reversibilidad es una premisa básica de la actividad científica. El slogan de hoy es "la flecha del tiempo"1. Incluso las partículas atómicas tienen un tiempo y cambian con el tiempo. Todo esto ha redundado en una modificación de las relaciones entre las ciencias sociales y las ciencias naturales.

Cuando yo era estudiante nos enseñaban que los científicos sociales éramos inferiores a los científicos de la naturaleza, pero que algún día nos hallaríamos a la par. Si perseverábamos, algún día podríamos hablar de los procesos sociales del modo que los naturalistas hablaban de los procesos físicos, esto es, que eran lineales, que tendían al equilibrio básico y que siendo irreversibles, las leyes que los rigen eran universales. $Y$ de pronto tenemos a un grupo mayoritario de científicos de la naturaleza diciéndonos: no, no, se trata en verdad de "la flecha del tiempo", de la flecha psicológica, pero el tiempo puede verse en otras direcciones ${ }^{2}$. Y ello significa, entre otras cosas, que las ciencias sociales y las naturales se van aproximando, pero ya no sobre la base del modelo newtoniano de la ciencia natural y de su mecanicismo, sino sobre la base de premisas que ya de antes eran fundamentales para las ciencias sociales. En efecto, el movimiento de dicho acercamiento va de las ciencias naturales a las ciencias sociales. En cierto modo lo que los físicos parecen estarnos diciendo es que son sociólogos inferiores análogamente a lo que decíamos cuando nos sentíamos menos científicos que ellos. En cualquier caso reconocen que los procesos sociales son los más complejos.

Y al mismo tiempo asistimos a un movimiento en las denominadas humanidades, que yo pienso tiene mucho que ver con los cambios en la política mundial y ha conducido a un auge de los estudios culturales. Los estudios culturales son un movimiento preponderante

\footnotetext{
${ }^{1}$ Wallerstein alude a uno de los capítulos de La historio riel Tiempo del físico y matemático inglés Stephen Hawking. en el que se puede leer: "El tiempo imaginario es indistinguible de las direcciones espaciales. Si uno puede ir hacia el norte, también puede dar la vuelta y dirigirse hacia el sur: de la misma Forma si uno puede ir hacia adelante en el tiempo imaginado debería poder también dar la vuelta e ir hacia atrás (...) las leyes de la ciencia no distinguen entre el pasado y el Futuro". Ver Stephen W. Hawking, Lo Historio del tiempo, Editorial Crítica, Barcelona, 1989, pp. 189-90. (Nota del traductor).

2 "Son la flecha termodinámica, la dirección en la cual el desorden aumenta; la flecha psicológica, la dirección del tiempo según la cual recordamos el pasado y no el futuro: y la flecha cosmogónica, la dirección del tiempo en la cual el universo se expande en vez de contraerse". Hawking, Opus nt, p. 200 (Nota del traductor). 
hoy en día. Su semillero fueron las humanidades, pero hoy hay muchos antropólogos e historiadores dedicados a ellos, y esa clase de estudios se está expandiendo hacia las demás ciencias sociales. Hay algo confuso todavía, pese a que la gente que se dedica a los estudios culturales le gusta acentuar el grado en que su enfoque es una reacción contra el cientificismo, e incluso una condena del mismo. Ellos se refieren desde luego al modelo newtoniano de ciencia, que como decía antes, ha sido abandonado por la propia ciencia natural. Pero lo que resulta impactante es el grado en que los estudios culturales significan un movimiento que, surgido en las humanidades, las va acercando progresivamente a las ciencias sociales. El objeto de los llamados estudios culturales es entendido como un proceso social más y por esa vía es una intersección entre las humanidades y las ciencias sociales. Tenemos entonces que no sólo las demarcaciones entre las ciencias sociales se están borrando, sino que la propia división tripartita humanidades, ciencias naturales, ciencias sociales- está siendo cuestionada.

\section{Un programa de reforma}

¿Qué clase de ciencia social debemos construir? Primero que todo, sugerimos que el problema del futuro no es simplemente una cuestión de reestructurar las ciencias sociales. Ni siquiera he sugerido que deba hacerse una. Lo que estoy diciendo es que el actual fundamento racional de las disciplinas ya no tiene mucho sentido. Y que mejor nos dediquemos a reflexionar sobre nuevos fundamentos racionales y sobre nuevos criterios de delimitación. Nótese que lo que hoy denominamos biología, hasta hace relativamente poco lo denominábamos zoología y botánica, y que hoy los departamentos de zoología y botánica virtualmente han desaparecido. La biología tiene muchas subdivisiones, pero la botánica y la zoología ya no son las divisiones en las cuales está organizada, luego el pastel puede dividirse de otras maneras.

Sugerimos que las universidades deben examinar la división tripartita. Está construida sobre el concepto de las "dos culturas" que se formuló para el siglo XVIII y que ha sido superado en gran medida. $Y$ no está mal que seamos un poco chovinistas y pensemos que las ciencias sociales puede ser centrales en el proceso en cuestión. Tenemos derecho a seguir pensando que la universidad sea el ámbito primario de la producción y reproducción del conocimiento. Hasta no hace mucho lo era. Con la formidable expansión de las universidades y de su población estudiantil, una de las cosas que ha ocurrido es lo que llamaría la gran escolarización del sistema universitario, esto es, la enorme presión social —uno tiene que enseñar cada vez más a un mayor número, y además seguir siendo relevante- una presión social para tener una gran número de graduados universitarios con la posibilidad de adquirir empleos profesionales, etc. Los profesores de secundaria se convierten en profesores de universidad, e incluso de posgrado, y están comenzando a ingresar a los institutos y centros avanzados. $Y$ tenemos que pensar lo que ocurrirá en 20 ó 50 años, si no desarrollamos instituciones más aptas, y si no solventamos el problema de sus bases financieras. ¿Cómo conseguiremos gente que haga investigación? Históricamente, la universidad ha sido la solución para el problema de financiar investigadores. Usted consigue trabajo como profesor, y por esa vía tiempo y eventualmente recursos para investigar y para hacer otras actividades académicas. Si la tendencia de ahora es empujar a los investigadores fuera de la Universidad, o ellos mismos se colocan fuera de ella, ¿quién y cómo se los financia?

Una recomendación específica de parte nuestra es que las Universidades y las instituciones afines estimulen algo que ya existe, aunque en pequeña escala: la posibilidad de que se formen grupos en torno a temas específicos durante un periodo de trabajo, un año digamos. Segundo, que en lugar de nuevos programas que se crean cada 
vez que alguien tiene la idea de trabajar sobre un tema $\mathrm{X}$, las universidades consideren la creación de un centro especialmente dedicado al tema por un período de digamos cinco años. Después ya se verá lo que pueden conseguir al término de ese período sin tener que ocuparse del problema de obtener fondos especiales. (Los fondos se asignarían desde el comienzo y no se gastaría tiempo elaborando propuestas para obtenerlos, que por experiencia es un gasto de tiempo poco retributivo). Ahora bien, mis dos sugerencias tienen, claro está, un costo. Pero tengo dos sugerencias adicionales, que a mi juicio son más importantes, y que, de sobremesa, no costarán un centavo.

Sugiero que a los profesores de las universidades se les nombre en cargos simultáneos. Hasta ahora ha habido una tendencia a favorecer personas relativamente distinguidas que rondan los cincuenta o sesenta años de edad. Cuando se trata de que esa persona encuentre atractivo la universidad, se le dice: "usted puede ser profesor de $X$ y Y simultáneamente". Pero es pura cortesía. El segundo cargo a menudo no tiene sentido, y realmente no se espera que haga nada allí. Sólo es un titulo honorífico que hace sentir bien al profesor. Deberíamos modificar esto totalmente. Nos gustaría decir: es obligatorio el doble compromiso. Ningún profesor en ninguna universidad pertenecerá a un solo departamento. Todos los profesores habrán de estar en dos. Cuando se habla en términos del primer departamento es aquel en el cual se tiene el $\mathrm{Ph}$. D. El segundo departamento podría ser cualquier otro. Y con el fin de impedir que los departamentos se resistan a ello, se insistirá que todos los departamentos deben tener al menos el $25 \%$ de sus profesores provenientes de algún otro de los que denominamos primarios. A mi juicio eso transformará a los departamentos. Puede inducir a nuevas combinaciones y no cuesta un centavo. En la medida en que se convierta en obligatoria, tal pauta funcionará. Cada profesor deberá estar adscrito a dos departamentos, pero él o ella, puede escoger el segundo. Y los departamentos deberán aceptar esta posibilidad, nadie podrá decir "aquí sólo aceptamos de los nuestros.

Lo mismo podría aplicarse para los estudiantes de posgrado. Hacer obligatorio que tomen un número de cursos en departamentos distintos a aquel que ofrece el programa. Por ahora ello es opcional, pero muchos departamentos se las arreglan para frustrar esa posibilidad. Ahora, en cambio, proponemos que usted no pueda obtener un título de Ph.D. en cualquier disciplina, a menos que tome la cuarta parte de los cursos en otro departamento. Obtendrá un título en la disciplina del departamento primario, pero también podrá escoger una segunda. Y los departamentos tendrán que adaptarse a eso.

Las anteriores son mis principales recomendaciones. Pienso que serán revolucionarias y subrayo que no se traducirán en incrementos presupuéstales, para concluir, permítanme citar la última frase de nuestro informe extenso: "lo más importante es que los aspectos subyacentes sean debatidos de modo claro, abierto, inteligente y de manera urgente". 